\title{
Grounds and limits: Reichenbach and foundationalist epistemology
}

\author{
Jeanne Peijnenburg • David Atkinson
}

Received: 13 May 2009 / Accepted: 8 June 2009 / Published online: 14 July 2009

(C) The Author(s) 2009. This article is published with open access at Springerlink.com

\begin{abstract}
From 1929 onwards, C. I. Lewis defended the foundationalist claim that judgements of the form ' $x$ is probable' only make sense if one assumes there to be a ground $y$ that is certain (where $x$ and $y$ may be beliefs, propositions, or events). Without this assumption, Lewis argues, the probability of $x$ could not be anything other than zero. Hans Reichenbach repeatedly contested Lewis's idea, calling it "a remnant of rationalism". The last move in this debate was a challenge by Lewis, defying Reichenbach to produce a regress of probability values that yields a number other than zero. Reichenbach never took up the challenge, but we will meet it on his behalf, as it were. By presenting a series converging to a limit, we demonstrate that $x$ can have a definite and computable probability, even if its justification consists of an infinite number of steps. Next we show the invalidity of a recent riposte of foundationalists that this limit of the series can be the ground of justification. Finally we discuss the question where justification can come from if not from a ground.
\end{abstract}

Keywords Foundationalism $\cdot$ Reichenbach $\cdot$ Probability

\section{Introduction}

In the debate between epistemic foundationalists and anti-foundationalists, Hans Reichenbach is clearly on the side of the latter. Already in his discussions with early logical positivists, Reichenbach's anti-foundationalist stance is noticeable: he rejects the positivistic idea that a theoretical sentence is equivalent to a set of observational sentences, arguing instead for a connection in terms of probability relations

\footnotetext{
J. Peijnenburg $(\varangle) \cdot$ D. Atkinson

Faculty of Philosophy, University of Groningen, Oude Boteringestraat 52,

Groningen 9712 GL, The Netherlands

e-mail: jeanne.peijnenburg@rug.nl
} 
(Reichenbach 1938). Reichenbach's anti-foundationalism is, however, most obvious in his debate with the American logician and epistemologist C. I. Lewis (1883-1964). The disagreement extended over more than two decades, from 1930 until 1952, and it is well-documented in letters and in contributions to journals.

Both Lewis and Reichenbach agree that epistemic justification is probabilistic in character: both believe that it makes sense to say that a proposition or a belief justifies another proposition or belief, even if the former does not logically imply the latter but merely gives probabilistic support. However, Lewis insists that probabilistic justification only makes sense if it springs from a ground that is not merely probable, but is certain. Reichenbach disagrees, holding that probabilistic justification remains coherent, even if it is not rooted in firm ground. In the present article we analyse the debate (Sects. 2-4), and explain its relevance to contemporary epistemology (Sects. 5-7).

We start in Sect. 2 by describing the basic difference between Lewis and Reichenbach. In Sect. 3 we explain Lewis's main argument for his claim that probability judgements presuppose certainties, namely that any regress of probability judgements, justified by probability judgements ad infinitum, has the absurd consequence of always yielding zero. Reichenbach explains to Lewis that this argument is flawed; but, as we will see, he is unable to convince his opponent. Rather, Lewis challenges Reichenbach to produce a counterexample, i.e., a particular regress of probability judgements that yields a number other than zero. Reichenbach never took up the challenge, but we will meet it in Sect. 4. By presenting a series that converges to a nonzero limit, we demonstrate that a probability judgement can have a definite, reasonable and computable value, even if its justification is infinitely postponed in the sense that it consists of an infinite number of steps. In this manner we show that Reichenbach, not Lewis, was correct, thereby also refuting later claims of Van Cleve (1977) and Legum (1980).

In Sects. 5 and 6 we discuss further the relevance of the Lewis-Reichenbach debate to present-day foundationalism. We first explain a recent riposte, which claims that foundationalism can be consistent with an infinite series of reasons. The riposte involves a particular example of a series which, although infinite, is claimed nevertheless to be foundational. We argue that the example is flawed for three reasons. First, its structure is quite different from a standard epistemic chain. Second, it is based on a confusion between the outcome of an infinite series and its origin. Third, and most seriously, the series in question implies that the probability of the target proposition is always one, independently of the nature and probability of the purported foundation.

Finally, in Sect. 7, we address the question whence epistemic justification can spring if not from a primordial source.

\section{Reichenbach versus Lewis}

In his first major work, Mind and the World-Order. An Outline of a Theory of Knowledge (1929), Lewis starts from the traditional view that our knowledge is partly mathematical and partly empirical. The mathematical part deals with knowledge that is a priori and analytic, the empirical part concerns our knowledge of nature. The crucial issue for any theory of knowledge, according to Lewis, is the character and the validity of empirical knowledge. Since "all empirical knowledge is probable only" (ibid., 
p. 309), the essential problem for a theory of knowledge is "that of the validity of our probability judgements" (ibid., p. 308).

A recurring theme in Mind and the World Order is that probability judgements only make sense if they are based on grounds that are certain:

The validity of probability judgements rests upon truths which must be certain. (ibid., p. 311)

...the immediate premises are, very likely, themselves only probable, and perhaps in turn based upon premises only probable. Unless this backward-leading chain comes to rest finally in certainty, no probability-judgment can be valid at all. (ibid., pp. 328-329)

From a letter that Lewis writes to Reichenbach on 26 August 1930, we can infer that Reichenbach had questioned these claims 1 month earlier, in a letter to Lewis that is now lost. Whatever the precise content of Reichenbach's letter may have been, it is clear that it did not convince Lewis. For 16 years later, in An Analysis of Knowledge and Valuation, Lewis stresses the same point again:

If anything is to be probable, then something must be certain. The data which themselves support a genuine probability, must themselves be certainties. (Lewis 1946, p. 186)

The disagreement between Lewis and Reichenbach reached its height in December 1951, when, at the 48th meeting of the Eastern Division of the American Philosophical Association at Bryn Mawr College, Reichenbach and Lewis both read papers relevant to this dispute. These papers were subsequently published in the The Philosophical Review of April 1952. In Lewis's contribution we read that he sticks to his guns:

The supposition that the probability of anything whatever always depends on something else which is only probable itself, is flatly incompatible with the assignment of any probability at all. (Lewis 1952, p. 173)

Reichenbach, as is clear, strongly disagrees with this foundationalist claim. Already in his major epistemological work, Experience and Prediction, he found an apt metaphor to summarize his own anti-foundationalist position:

All we have is an elastic net of probability relations, floating in open space. (Reichenbach 1938, p. 192)

Lewis's claim that this is not so, and that probabilities must be grounded in certainties, is called by Reichenbach "just one of those fallacies in which probability theory is so rich" (Reichenbach 1952, p. 152). In an attempt to understand the root of the fallacy Reichenbach writes:

We argue: if events are merely probable, the statement about their probability must be certain, because ...Because of what? I think there is tacitly a conception involved according to which knowledge is to be identified with certainty, and probable knowledge appears tolerable only if it is embedded in a framework of certainty. This is a remnant of rationalism. (Reichenbach 1952, p. 152) 
Lewis, in turn, rejects the accusation of being an old fashioned rationalist and replies that, on the contrary, he is trying to save empiricism from what he calls a modernized coherence theory like that of his opponent (Lewis 1952, pp. 171, 173). He writes:

...[Reichenbach's] probabilistic conception strikes me as supposing that if enough probabilities can be got to lean against one another they can all be made to stand up. I suggest that, on the contrary, unless some of them can stand up alone, they will all fall flat. (Lewis 1952, p. 173)

In an attempt to arbitrate the matter, we will in Sect. 3 look at the argument with which Lewis defends his claim that probabilities presuppose grounds that are certain.

\section{Lewis's argument}

Lewis's argument, as it appears in Mind and the World Order, is the following:

Nearly all the accepted probabilities rest upon more complex evidence than the usual formulations suggest; what are accepted as premises are themselves not certain but highly probable. Thus our probability judgement, if made explicit, would take the form: the probability that $A$ is $B$ is $a / b$, because if $P$ is $Q$, then the probability that $A$ is $B$ is $m / n$, and the probability of ' $P$ is $Q$ ' is $p / q$ (where $m / n \times p / q=a / b)$. But this compound character of probable judgement offers no theoretical difficulty for their validity, provided only that the probability of the premises, when pushed back to what is more and more ultimate, somewhere comes to rest in something certain. (Lewis 1929, pp. 327-328)

So Lewis's argument amounts to this. He first stresses that the statement 'the probability that $A$ is $B$ is $a / b$ ' or

$$
P\left({ }^{\prime} A \text { is } B '\right)=a / b
$$

is elliptical for 'the probability that $A$ is $B$ is $m / n$, if $P$ were $Q$, but the probability that $P$ is $Q$ is $p / q$ '. In symbols:

$$
\begin{aligned}
P\left({ }^{\prime} A \text { is } B^{\prime}\right) & =P\left({ } ^ { \prime } A \text { is } B ^ { \prime } | ' P \text { is } Q \text { ') } P \left({ }^{\prime} P \text { is } Q\right.\right. \text { ') } \\
& =(m / n) \times(p / q) \\
& =a / b .
\end{aligned}
$$

Now of course the probability that $P$ is $Q$ may also be elliptical. If this series were to go on and on, then, because all the factors in the multiplication are probabilities (positive numbers less than one), the probability of the original proposition ' $A$ is $B$ ' would always tend to zero. But this is ridiculous, so the series of probability judgements must come to a stop in a statement that is certain. 
Lewis's argument is, however, simply mistaken. For $P$ (' $A$ is $B$ ') is not elliptical for the product $\mathrm{P}$ (' $A$ is $B$ ' ' $P$ is $Q$ ') $P$ (' $P$ is $Q$ '), but is elliptical for the sum of products

$$
\begin{aligned}
& P\left({ }^{\prime} A \text { is } B{ }^{\prime} \mid{ }^{\prime} P \text { is } Q \text { ') } P\left({ }^{\prime} P \text { is } Q '\right)\right. \\
& +P\left({ }^{\circ} A \text { is } B^{\prime} \mid \text { not-' } P \text { is } Q \text { ') } P \text { (not-' } P \text { is } Q\right. \text { '). }
\end{aligned}
$$

Lewis forgets that, if the probability of ' $A$ is $B$ ' is conditioned by the probability of ' $P$ is $Q$ ', then you can only calculate the probability of ' $A$ is $B$ ' if you also take into account what the probability of ' $A$ is $B$ ' is in case ' $P$ is $Q$ ' is false.

Almost 20 years later, Bertrand Russell also champions the Lewisian claim that probabilities require grounds that are certain. It is interesting, to say the least, that he defends this claim with exactly the same erroneous argument that Lewis had used (Russell 1948 , pp. 433-435). Reichenbach notices this and he explains it to Russell in a letter on 28 March 1949 (Reichenbach and Cohen 1978, pp. 405-411). Three weeks later, on the 22nd of April, Russell sends a reply in which he acknowledges his mistake.

Lewis, however, seems to have persisted in the error of his ways, and Reichenbach confronts him with this fact in 1951, at the 48th meeting of the APA at Bryn Mawr. Lewis appears however not to be impressed by Reichenbach's amendment. Apparently failing to see the relevance of the second term in (3), he simply states:

I disbelieve that this will save [Reichenbach's] point. For that, I think he must prove that, where any regress of probability-values is involved, the progressively qualified fraction measuring the probability of the quaesitum will converge to some determinable value other than zero; and I question whether such a proof can be given. (Lewis 1952, p. 172)

In other words, Lewis simply does not believe that an infinite regress of probabilities can converge to some value other than zero. Even if we do take Reichenbach's amendment into account, then for Lewis it is still the case that an infinite series of probability statements conditioned by probability statements will always converge to zero. And he defies Reichenbach to prove the contrary. Reichenbach never took up the challenge, but in the next section we will do so, as it were on Reichenbach's behalf.

\section{Meeting Lewis's challenge}

Reichenbach in fact corrects Lewis and Russell by citing a theorem that follows directly from the probability axioms. For Eq. 3 is an instance of the rule of total probability:

$$
P\left(E_{0}\right)=P\left(E_{0} \mid E_{1}\right) P\left(E_{1}\right)+P\left(E_{0} \mid \neg E_{1}\right) P\left(\neg E_{1}\right),
$$

where $E_{0}=$ ' $\mathrm{A}$ is $\mathrm{B}$ ' and $E_{1}=$ 'P is Q'. If $E_{1}$ itself is only probable, grounded in $E_{2}=$ ' $\mathrm{R}$ is $\mathrm{S}$ ', then formula (4) must be iterated, and this produces a much more complicated regression than the simple product that Russell and Lewis had envisaged. 
Below we will give an example in which Eq. 4 is iterated infinitely many times. We will show that, even when the iteration is infinite, it need not converge to zero. ${ }^{1}$

Let $P\left(E_{0}\right)$ in Eq. 4 be the probability that a man will have a certain hereditary disorder. Let $P\left(E_{0} \mid E_{1}\right)$ be the conditional probability that he will have the complaint, given that his father had it. Under the assumption that not all people with this disorder have fathers with a similar affliction, $P\left(E_{0} \mid \neg E_{1}\right)$ is not zero. On the other hand, we know that a man is more likely to contract the disorder if his father had it than if he did not. That means that $1>P\left(E_{0} \mid E_{1}\right)>P\left(E_{0} \mid \neg E_{1}\right)>0$, and we may assume that approximate empirical values of these two probabilities have been estimated from the study of large populations. With $\alpha=P\left(E_{0} \mid E_{1}\right)$ and $\beta=P\left(E_{0} \mid \neg E_{1}\right)$, we may rewrite formula (4) in the form

$$
\begin{aligned}
P(E) & =\alpha P\left(E_{1}\right)+\beta\left(1-P\left(E_{1}\right)\right) \\
& =\alpha P\left(E_{1}\right)+\beta-\beta P\left(E_{1}\right) \\
& =\beta+(\alpha-\beta) P\left(E_{1}\right) .
\end{aligned}
$$

$P\left(E_{1}\right)$ is the probability that the father had the disorder, and of course this probability can be in turn conditioned by the fact that his father did, or did not similarly suffer. Thus we have

$$
P\left(E_{1}\right)=\beta+(\alpha-\beta) P\left(E_{2}\right),
$$

where $P\left(E_{2}\right)$ is the probability that the man's paternal grandfather contracted the infirmity. Of course, the value of $P\left(E_{2}\right)$ is calculated in the same way, giving:

$$
P\left(E_{2}\right)=\beta+(\alpha-\beta) P\left(E_{3}\right),
$$

where $P\left(E_{3}\right)$ is the probability that the man's paternal great-grandfather contracted it. After $n$ steps we have:

$$
\begin{aligned}
P\left(E_{0}\right)= & \beta+\beta(\alpha-\beta)+\beta(\alpha-\beta)^{2}+\cdots \\
& +\beta(\alpha-\beta)^{n}+(\alpha-\beta)^{n+1} P\left(E_{n+1}\right) .
\end{aligned}
$$

The remainder term in Eq. $6,(\alpha-\beta)^{n+1} P\left(E_{n+1}\right)$, is the product of two factors. The factor $P\left(E_{n+1}\right)$ is the probability that the primal grandfather had the disease. The other factor, $(\alpha-\beta)^{n+1}$, gets smaller as $n$ gets bigger, and in the limit that $n$ goes to infinity, $(\alpha-\beta)^{n+1}$ tends to zero. But if $(\alpha-\beta)^{n+1}$ tends to zero, the entire remainder term dwindles away to nothing. In other words, with an infinite number of steps, the remainder term vanishes and what remains is the infinite series

\footnotetext{
${ }^{1}$ See also Sect. 4 of Atkinson and Peijnenburg (2006) .
} 


$$
\begin{aligned}
P\left(E_{0}\right) & =\beta+\beta(\alpha-\beta)+\beta(\alpha-\beta)^{2}+\ldots \\
& =\beta\left[1+(\alpha-\beta)+(\alpha-\beta)^{2}+\ldots\right] \\
& =\beta \sum_{n=0}^{\infty}(\alpha-\beta)^{n} .
\end{aligned}
$$

Since $0<\alpha-\beta<1$, this geometric series is convergent and its sum is

$$
P\left(E_{0}\right)=\frac{\beta}{1-\alpha+\beta} .
$$

And this is certainly not zero. For example, if $\alpha=0.8$ and $\beta=0.05$, then the value of $P\left(E_{0}\right)$ is 0.2 . Conclusion: Lewis is mistaken. It is not the case that an infinite series of probability statements conditioned on probability statements always tends to zero-we have just seen an example of such a series that does not do so. ${ }^{2}$

\section{A foundationalist reply}

Contemporary foundationalists are of course very different from Lewis, yet they resemble him in some of their views. For example, like Lewis many have a probabilistic view of epistemic justification. They believe that it makes sense to say that $E_{n+1}$ justifies $E_{n}$ even if the former does not logically imply the latter; it is enough if $E_{n+1}$ only probabilistically supports $E_{n}$ (with respect to some measure and with some degree of epistemic support). Moreover, like Lewis, they feel uneasy when confronted by a series in which the epistemic support is continued ad infinitum, never reaching firm ground. Presumably most of them will not fall into the trap of thinking that an infinite regress of probability values must yield zero, but they do have the feeling that such a regress is incoherent in one way or another. And this feeling should of course not come as a surprise. After all, what could be more central to foundationalism than the claim that there has to be a foundation, a last member that serves as the basis of the entire edifice, the source from which the whole justification springs? This foundation may be a certainty, as it is in Lewis's philosophy, or a fixed probability, as it is for moderate foundationalists, or a probability that is not fixed, as it is for so-called weak foundationalists (Bonjour 1985). But however he twists and turns, a genuine foundationalist seems attached to a foundation, a last link in the chain.

Nevertheless there have recently been foundationalists who argue that an epistemic chain need not have a last link. John Turri, for example, writes:

A series of reasons, supporting a belief in a questioned non-evident proposition, need not have a last member in order to have a foundational (properly basic) member. ...Foundationalism is consistent with there being available ....an infinite, non-repeating series of reasons, of the sort the infinitist prizes. In other

\footnotetext{
2 The objection that the series in our example is not really infinite, because it starts off with a primal grandfather, begs the question. For a primal grandfather does not enter the scene as a deus ex machina: he has ancestors too, in the form of primates, vertebrates, invertebrates, etc., eventually going back to the Big Bang. And the question is precisely whether that was the beginning, indeed whether there was a beginning.
} 
words, foundationalists needn't limit themselves to a finite series of reasons. (Turri 2009, pp. 162-163)

Here is Turri's example of an infinite series that he claims to be compatible with foundationalism:

Suppose that Fran sees that it is 2:05. Suppose further that she practices a version of foundationalism according to which such external-world beliefs can be properly basic, and that in the present case her belief that it is 2:05 satisfies all the relevant criteria. Now Fran asserts that it is past 2:00 ... She ...believes it is past 2:00 because it is past 2:04 ...why think that it is past 2:04? Because it is past 2:04:30. Why think that? Because it is past 2:04:45 ... But proceeding this way ensures that she will approach the limit of, but never arrive at, 2:05. In other words, she has available to her an infinite series of non-repeating reasons, each of which is entailed by its successor. Moreover, the foundationalist has a principled story to tell about how each member of this infinite series gets justified for her: namely she can see that it is 2:05! (ibid., p. 163)

In other words, Turri claims that the limit serves as the ground of the infinite and convergent series. Is that tenable?

\section{Limits as grounds?}

There are three things that should be noted about Turri's example. The first is that it is not a standard epistemic chain. When infinite, a standard epistemic chain has the form

$$
E_{0} \longleftarrow E_{1} \longleftarrow E_{2} \longleftarrow E_{3} \ldots \ldots \infty
$$

where each link $E_{n}$ is justified by $E_{n+1}$. Turri's idea is that the whole infinite chain is supported by the basic belief $\Omega$, and thus has the form

$$
E_{0} \longleftarrow E_{1} \longleftarrow E_{2} \longleftarrow E_{3} \ldots \ldots \longleftarrow \Omega
$$

But in fact the structure of Turri's example is quite different. It may be pictured as follows:

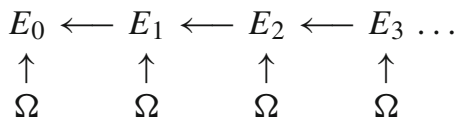

where $\Omega$ is Fran's basic belief "It is 2:05", and where

$E_{0}$ : "It is past 2:00"

$E_{1}$ : "It is past 2:04"

$E_{2}$ : "It is past 2:04:30"

$E_{3}$ : "It is past 2:04:45" 
and so on. In (11), each $E_{n}$ is entailed by $E_{n+1}$, just as was the case in the array (10). In contrast to (10), however, in (11) $\Omega$ directly entails each of the $E_{n}$ separately; rather than an epistemic chain, (11) constitutes an infinite number of single implications, all having the same antecedent, $\Omega$. The fact that each $E_{n}$ is also entailed by $E_{n+1}$ does not add anything at all to its credibility.

The second remark to be made about Turri's example concerns his use of the term 'limit'. Normally, the limit denotes the value or outcome of a series when its length grows beyond all bounds, not its origin. In casu this refers to the probability of the non-evident target proposition $E_{0}$. In the Fran example, however, Turri tries to identify the limit with the alleged origin of the series, namely $\Omega$. He thereby confuses the result of an infinite series with its supposed initiator.

The third and final thing we may note about the Fran example is that $\Omega$ entails each $E_{n}$, which is more than merely making each $E_{n}$ more probable. Similarly, the relation between $E_{n+1}$ and $E_{n}$ is that of logical implication rather than of probabilistic support. With the notation $\alpha_{n}=P\left(E_{n} \mid E_{n+1}\right)$ and $\beta_{n}=P\left(E_{n} \mid \neg E_{n+1}\right)$, we have $\alpha_{n}=1$, since $E_{n}$ is entailed by $E_{n+1}$. However, $\beta_{n}$ could be nonzero. After all, $E_{n}$ can be true when $E_{n+1}$ is false. If it were not the case that $E_{n}$ could be true when $E_{n+1}$ is false, the structure would not be that of entailment, but bi-entailment. We would not just have $E_{n+1} \longrightarrow E_{n}$, but also $E_{n} \longrightarrow E_{n+1}$. Such bi-entailment would be equivalent to having $\alpha_{n}=1$ and $\beta_{n}=0$.

Be that as it may, in Turri's scenario it is certainly the case that $\beta_{n}$ is not zero. Take for example $\beta_{0}=P\left(E_{0} \mid \neg E_{1}\right)$. If $E_{1}$ were false, i.e., if it were not the case that is past 2:04, $E_{0}$ could still be true, for it might be 2:02, for example, and then it would indeed be past 2:00. So $\beta_{0}$ is greater than zero, and the same goes for each of the $\beta_{n}$. However, when $\alpha_{n}=1$ and $\beta_{n}>0$, there are serious consequences if the chain is of infinite length. For an infinite chain will then always lead to the conclusion that the target belief $E_{0}$, or in Turri's words the "belief in a questioned non-evident proposition", has probability unity. This in itself is not bad news for Turri, for he works after all with implication, and not with probabilistic support. What is, however, disastrous for his claim is that $P\left(E_{0}\right)=1$ quite independently of what $\Omega$ is. Indeed, even if $\Omega$ were replaced by not- $\Omega=$ 'It is not past 2:05', the infinite chain would still lead to certainty. This can be further elucidated as follows.

Appealing once more to the rule of total probability, now in the form

$$
P\left(E_{n}\right)=P\left(E_{n} \mid E_{n+1}\right) P\left(E_{n+1}\right)+P\left(E_{n} \mid \neg E_{n+1}\right) P\left(\neg E_{n+1}\right),
$$

we find that

$$
P\left(\neg E_{n}\right)=P\left(\neg E_{n} \mid \neg E_{n+1}\right) P\left(\neg E_{n+1}\right),
$$

with use of $P\left(E_{n} \mid E_{n+1}\right)=1 .{ }^{3}$ The relation (12) can be iterated from $n=0$ to $n=s$ :

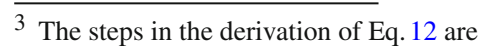

$$
\begin{aligned}
P\left(E_{n}\right) & =P\left(E_{n+1}\right)+P\left(E_{n} \mid \neg E_{n+1}\right) P\left(\neg E_{n+1}\right) \\
1-P\left(E_{n}\right) & =1-\left[P\left(E_{n+1}\right)+P\left(E_{n} \mid \neg E_{n+1}\right) P\left(\neg E_{n+1}\right)\right]
\end{aligned}
$$




$$
P\left(\neg E_{0}\right)=\left\{\prod_{n=0}^{s} P\left(\neg E_{n} \mid \neg E_{n+1}\right)\right\} P\left(\neg E_{s+1}\right) .
$$

What happens as we take $s$ to infinity? $P\left(E_{n} \mid \neg E_{n+1}\right)$ is not 0 , as we noted, therefore, $P\left(\neg E_{n} \mid \neg E_{n+1}\right)$ is a positive number less than 1 , since the sum of $P\left(E_{n} \mid \neg E_{n+1}\right)$ and $P\left(\neg E_{n} \mid \neg E_{n+1}\right)$ equals 1 . When $s$ in (13) tends to infinity, the product of factors between the parentheses has an infinite number of terms, each less than one. In this limit the product will normally be zero. But of course, this means that the whole right hand side of (13) equals zero. From this it follows that $P\left(\neg E_{0}\right)=0$ and thus that $P\left(E_{0}\right)=1.4$

Far from supporting Turri's hope that his infinite chain can serve the foundationalist's purpose, the above reasoning has provided ammunition for an infinitist new-style (see Peijnenburg 2007). For, in complete accordance with the Weltanschauung of the latter, we have here a proposition that is entailed (up to measure zero) by an infinite chain of conditional probabilities. ${ }^{5}$ No anchoring of the infinite sequence of propositions to a basic belief is required. Nor is it possible, as we have just shown.

\section{Where then does the justification come from?}

If the justification of a target proposition by means of an infinite regress of epistemic support does not come from a basic belief, where does it come from? How can we make sense of the idea that there can be justification without a ground?

The answer is that the justification by an infinite chain comes entirely from the conditional probabilities that constitute its links. We do not need a single source from which justification springs in order to have justificatory relations between beliefs or propositions. Recall the manner in which we (probabilistically) justified a non-evident target proposition $E_{0}$ : we calculated its unconditional probability on the basis of a series of conditional probabilities. If the series is finite, then there is a last link, a basic belief that constitutes the starting point of the chain. Part of the justification comes from this basic belief and part comes from the conditional probabilities that connect the basic belief to the target proposition. If the chain is very long but still finite, the

Footnote 3 continued

$$
\begin{aligned}
& =1-P\left(E_{n+1}\right)-P\left(E_{n} \mid \neg E_{n+1}\right) P\left(\neg E_{n+1}\right) \\
P\left(\neg E_{n}\right) & =P\left(\neg E_{n+1}\right)-P\left(E_{n} \mid \neg E_{n+1}\right) P\left(\neg E_{n+1}\right) \\
& =\left[1-P\left(E_{n} \mid \neg E_{n+1}\right)\right] P\left(\neg E_{n+1}\right) \\
& =P\left(\neg E_{n} \mid \neg E_{n+1}\right) P\left(\neg E_{n+1}\right) .
\end{aligned}
$$

4 In general a product like the one in Eq. 13 could fail to tend to zero in the limit that $s$ tends to infinity, but only if the conditional probabilities tend very rapidly to unity as $n$ tends to infinity. In the normal case, however, and certainly if the conditional probabilities have a uniform upper bound that is less than 1 , the infinite product is zero.

5 A special case of this result can be obtained from the example (7), in which the conditional probabilities were the same from step to step of the iteration. It suffices to set $\alpha=1$ in Eq. 8 to obtain $P\left(E_{0}\right)=1$, on condition that $\beta \neq 0$. 
justificatory role of the basic belief diminishes while the combined contribution of the conditional probabilities to the justification increases. The longer the chain becomes, the less the justification by the basic belief and the more the justification provided by the conditional probabilities. This is no surprise, for the conditional probabilities do not arise out of thin air: they are part and parcel of the epistemic chain. If the series is infinite (and convergent), then all of the justification is carried by the conditional probabilities, and none by the basic belief. Indeed, the basic belief has then become completely irrelevant.

It is instructive to illustrate our answer by means of another example. Consider an inheritable trait, $\mathrm{T}$, conducive to survival in a particular environment, which a girl is sure to have if her mother had it. Suppose though that the child might also carry $\mathrm{T}$ if her father had it, whether or not mother did so. Thus the probability that the child has $\mathrm{T}$, given that mother has $\mathrm{T}$, is 1 ; but the probability that she has $\mathrm{T}$ if mother lacks $\mathrm{T}$ is not zero, since there is a chance after all that father has $\mathrm{T}$ (assuming that there is no other way that the child can get T). In terms of our symbolism, this situation satisfies $\alpha_{n}=P\left(E_{n} \mid E_{n+1}\right)=1$ and $\beta_{n}=P\left(E_{n} \mid \neg E_{n+1}\right)>0$, where $E_{n}$ is the proposition that a female in the $n$th generation carries T, and $E_{n+1}$ is the proposition that her mother has T. So the structure of the example is formally the same as it was in Turri's example. ${ }^{6}$

The new example has the advantage that further investigation will yield insight into the origin of the justification of the claim that a given girl has the inheritable trait, as symbolized by $P\left(E_{0}\right)=1$. The probability that a girl has $\mathrm{T}$ is greater than the probability that mother had $\mathrm{T}$ (on condition that the latter probability is not 1 ) simply because there is a nonzero probability that father carries $\mathrm{T}$. The probability that the child has $\mathrm{T}$ is even greater than the probability that her maternal grandmother had $\mathrm{T}$, since the possibilities are more numerous: there are after all three other grandparents, each of whom could be a T-carrier. It is intuitively clear-reaching further and further back into the family tree - that the genetic condition of a great-great-grandmother in the $n$th ancestral generation contributes less and less, as $n$ increases, to the probability that the girl has T. In the formal limit of an infinite number of generations, all the contributions to the probability that the child has $\mathrm{T}$ are coming from the conditional probabilities: the contribution of a remote ancestress diminishes more and more as the ancestress is further and further away, until she is hidden in the mists of time and her influence has vanished completely. ${ }^{7}$

A final worry seems to remain. If a basic belief about a primal grandfather or a first ancestress has no influence on the probability of our target proposition, then how could our chain of reasons apply to the world? Are we not, in concluding that all epistemic support comes from conditional probabilities, detaching our epistemic chain from the real world around us? And if so, how can we distinguish our reasonings from those

\footnotetext{
6 In Turri's example there is of course also $\Omega$, which is absent here; but, as we have explained, in infinite series the justificatory role of basic beliefs such as $\Omega$ is nihil.

7 The fact that the child is sure to carry $\mathrm{T}$, in the case that the number of generations is infinite, is a consequence of the assumption that it is certain that she will carry $\mathrm{T}$ if her mother does so. If this conditional probability is reduced, the unconditional probability that the child carries T is also reduced (cf. Eq. 8 for the case that the conditional probabilities are the same from generation to generation).
} 
occurring in fiction, in the machinations of a liar, or in the hallucinations of a heroin addict? In terms of our example: how can we ever distinguish the story about a real trait $\mathrm{T}$ from a fairy tale with the same structure in which, instead of $\mathrm{T}$, there is an inheritable magical power $\mathrm{M}$ to turn a prince into a frog?

The distinction is not far to seek. It lies in the mundane fact that in the former, but not in the latter, the conditional probabilities connect to that possible world which is the actual world. Research in many empirical case studies might have established that all T-carriers had at least one parent who carried T. In the fairy tale, on the other hand, the only 'evidence' that $\mathrm{M}$ is inheritable is contained in the story itself-outside the tale there is no evidence at all. We realise that this answer will not convince the confirmed sceptic, but our opponent after all is the foundationalist, not the sceptic. To our friend the foundationalist we say: the fact that conditional probabilities are connected to the real world does not imply that they must have an origin in the sense of a basic belief. An infinite series of conditional probabilities can refer to the real world without their being tied to an unconditional probability. Some foundationalists might not be convinced, objecting that an appeal to notions like 'as applying to the real world', 'outside evidence' and 'empirical case studies' only makes sense within a framework of foundationalism. But if all that is left of foundationalism is the acceptance of estimated empirical conditional probabilities, then perhaps even Reichenbach might have joined the foundationalist club.

Open Access This article is distributed under the terms of the Creative Commons Attribution Noncommercial License which permits any noncommercial use, distribution, and reproduction in any medium, provided the original author(s) and source are credited.

\section{References}

Atkinson, D., \& Peijnenburg, J. (2006). Probability without certainty. Studies in History and Philosophy of Science, 37, 442-453.

Bonjour, L. (1985). The structure of empirical knowledge. Cambridge, MA: Harvard University Press.

Lewis, C. I. (1929). Mind and the world-order. An outline of a theory of knowledge. New York: C. Scribner's Sons.

Lewis, C. I. (1946). An analysis of knowledge and valuation. La Salle, IL: Open Court.

Lewis, C. I. (1952). The given element in empirical knowledge. The Philosophical Review, 61(2), 168-172. Legum, R. A. (1980). Probability and foundationalism. Philosophical Studies, 38(4), 419-425.

Peijnenburg, J. (2007). Infinitism regained. Mind, 116, 597-602.

Reichenbach, H. (1938). Experience and prediction. Chicago: University of Chicago Press.

Reichenbach, H. (1952). Are phenomenological reports absolutely certain? The Philosophical Review, 61(2), 147-159.

Reichenbach, M. \& Cohen, R. (Eds.). (1978). Hans Reichenbach. Selected writings 1909-1953. Dordrecht: Reidel.

Russell, B. (1948). Human knowledge. London: George Allen and Unwin.

Turri, J. (2009). On the regress argument for infinitism. Synthese, 166(1), 157-163.

Van Cleve, J. (1977). Probability and certainty. Philosophical Studies, 32(4), 323-334. 